\title{
Entre la inmutabilidad y el cambio: una lectura de L'anté- peuple de Sony Labou Tansi
}

Recibido: 20 de octubre, 2020. Aceptado: 27 de abril, 2021.

Por: Mg. Oscar Iván Arcos Guerrero', Universidad Nacional de José Clemente Paz, Argentina, ORCID: 0000-0002-2235-4585.

\section{Resumen}

Este artículo inicia exponiendo el argumento de L'anté-peuple, novela publicada por Sony Labou Tansi en 1983. Sobre esa base, presenta la tesis que defiende el autor del estudio a lo largo de estas páginas: la obra analizada da cuenta de la inmutabilidad de las condiciones político-sociales vividas tanto en el Zaire como en la República Popular del Congo y también revela la transformación experimentada por sus personajes. La particularidad de L'anté-peuple con respecto al resto de novelas del Labou Tansi es lo que suscita el conjunto de observaciones que se realizan en este trabajo, pues es una ficción que tiene un tono más personal y, al mismo tiempo, más distante de los paradigmas estéticos dominantes en su época que aquel que poseen las demás novelas del autor elegido. El artículo se divide en dos partes: la primera se ocupa de mostrar aspectos generales de la historia de los países mencionados para luego observar la manera en la que el autor los reelabora en su ficción; la segunda, en cambio, muestra a los personajes en tanto entidades ficcionales que cambian con el transcurrir de los acontecimientos de los cuales forman parte. Ambos apartados permiten arribar a las conclusiones que aparecen al final del texto, en las cuales se presenta L'anté-peuple como una producción literaria cuya estética resulta compleja por la manera en la que interactúan entre sí los aspectos de la ficción abordados en el análisis.
Oscar Iván Arcos Guerrero. Entre la inmutabilidad y el cambio: una lectura de L'anté-peuple de Sony Labou Tansi. Revista Comunicación. Año 42, volumen 30, número 1, enero-junio, 2021. Instituto Tecnológico de Costa Rica. ISSN: 0379-3974 / e-ISSN1659-3820.

1 El profesor Oscar Iván Arcos Guerrero estudió Español y Lenguas Extranjeras en la Universidad Pedagógica Nacional de Colombia. Es Magíster en Literaturas en Lenguas Extranjeras y en Literaturas Comparadas de la Universidad de Buenos Aires. Actualmente, cursa estudios de doctorado en el Instituto de Literatura Hispanoamericana de la última casa de estudios mencionada, gracias a la beca otorgada por el Consejo Nacional de Investigaciones Científicas y Técnicas de Argentina (CONICET). Desde el 2015, labora como docente en la Universidad Nacional de José Clemente Paz, Argentina. Contacto: oguerrero@unpaz.edu.ar.

PALABRAS CLAVE:

Literatura, África al Sur del Sahara, Zaire,

Congo, opresión, cambio cultural

\section{KEY WORDS:}

Literature, Sub-Saharan Africa, Zaire, Congo, oppression, cultural change 


\section{Abstract \\ Between immutability and change: a reading of Sony Labou Tansi's L'anté-peuple}

This article begins by exposing the plot of Sony Labou Tansi's novel L'anté-peuple, published in 1983. In this study, the author contends that the analyzed work accounts for the immutability of the politico-social conditions experienced both in Zaire and in the People's Republic of Congo and also reveals the transformation experienced by its characters. L'anté-peuple is unique among the rest of Labou Tansi's novels, as it is a fiction of a more personal nature and, at the same time, it is also narrated in a more distant tone of the dominant aesthetic paradigms of its time. The first section of this article lays out general aspects of the historical events of both countries, delving into the way in which the author reworks them in his fiction; the second one shows how the fictional characters change as events unfold. Both sections lead to the conclusions at the end of the text. In the final part of this article, its autor asserts that the analysis of the interacting aspects of fiction in L'antépeuple shows it is as a literary production of complex aesthetics.

El argumento podría resumirse de la siguiente forma: un hombre cuyo trabajo como director de un instituto pedagógico le brinda una posición política y social reconocida es seducido por una de las estudiantes que pertenecen al centro de educación que tiene a cargo. Evita a toda costa ceder ante el deseo y la tentación que le produce la alumna, refugiándose incluso en el alcohol. De pronto, se entera de que ella muere y de que deja una carta que lo acusa de su deceso. La carta lo incrimina. A partir de ello, el mundo que lo rodea se desmorona; comienza una nueva etapa en su vida que inicia con la reclusión en prisión. Ahí se da cuenta del grado de corrupción que ha alcanzado el gobierno de su país y de la imposibilidad de salir del problema en que se encuentra por las vías legales. Con la ayuda de algunas personas, logra escapar de su celda y cruzar la frontera fluvial que lo separa de su exilio. Una vez en el extranjero, se entera de que las cosas no difieren mucho de cómo son en su patria: hay detenciones, abusos de poder por parte de las fuerzas públicas y temor en toda la población. El hombre en cuestión es víctima de las persecuciones y desapariciones llevadas a cabo por los militares. Algunos compañeros de ruta — también exiliados- se lanzan a su búsqueda; otros, por azares del destino, caen asimismo en los allanamientos realizados. Con todo, el hombre y la persona que más lo estima se integran a la milicia y contribuyen a la lucha política que se libra contra el régimen.
Contada de este modo, tal historia no nos es del todo ajena a quienes la leemos desde América Latina: los conflictos dictatoriales vividos por diversos países de nuestra región en distintas épocas y las implicaciones que tuvieron para nuestra sociedad nos permiten establecer cierto grado de reconocimiento con la trama que se teje. Sin embargo, pertenece a una de las tradiciones literarias configurada al otro lado del Atlántico: la del África subsahariana de expresión francesa. L'anté-peuple (1983), novela de Sony Labou Tansi traducida al español en 2010 por Manuel Serrat Crespo para El Aleph Editores, narra la desventura sufrida por Nitu Dadou, personaje ficcional que debe abandonar Kinshasa, capital de Zaire, para buscar refugio en Brazzaville, capital de la República Popular del Congo. El mismo año de su publicación, el libro gana el Gran Premio Literario del África Negra (Makita, 1997), lo cual permite pensar en su consagración dentro del ámbito literario de su región. De ahí que la novela merezca el despliegue de algunas páginas para su interpretación que permitan apreciar su valor literario, pues muestra al lector la inmutabilidad de las condiciones político-sociales a ambos lados del río Congo y la transformación experimentada por sus personajes. Así, tal obra reelabora, desde la ficción, los avatares que trajeron consigo las independencias para los jóvenes países africanos que hemos mencionado y para sus ciudadanos.

La tesis que acabamos de formular toma en cuenta los planteamientos de Chimamanda Ngozi Adichie (2009), en tanto busca alejarse de la concepción de 
África como un espacio geo-cultural con una única historia. Consideramos que tal continente, al igual que América Latina, ha sido reducido a los estereotipos que circulan en las grandes metrópolis del mundo. En ese sentido, nuestro trabajo se limita al estudio de la novela de Labou Tansi que hemos elegido y a analizar cómo se observan en ella algunos aspectos de su cultura. Por lo demás, vale la pena mencionar que las páginas que aquí presentamos son producto de las reflexiones derivadas del proyecto de investigación doctoral que actualmente realizamos en el Instituto de Literatura Hispanoamericana de la Universidad de Buenos Aires, gracias a la beca otorgada por el Consejo Nacional de Investigaciones Científicas y Técnicas de Argentina (CONICET). Tal proyecto se titula "América Latina y el África francófona: las poéticas de Borges y García Márquez en diálogo con las novelas de Sony Labou Tansi, Rachid Boudjedra y Tahar Ben Jelloun".

Ahora bien, la importancia de nuestro estudio radica en la singularidad que L'anté-peuple supone dentro del conjunto de novelas de Labou Tansi: escrita antes de La Vie et demie (1979) pero publicada con posterioridad a esta última, la ficción que nos ocupa posee un tono más personal y, a su vez, más alejado de los cánones estéticos imperantes en la época que el que tiene el resto de su producción novelística. Así, las demás novelas publicadas por el mismo autor se enmarcan, en términos generales, dentro de una misma corriente: aquella correspondiente a la escritura barroca e incluso carnavalesca. Para ello, toma prestados muchos aspectos de los modelos latinoamericanos del momento, lo cual le permite satisfacer las expectativas que algunos medios culturales instaurados en París tenían respecto a la identidad, a la literatura y a la modernidad africanas (Devesa, 1996). Tanto el trabajo de Devesa (1996) como el de Makita (1997) que empleamos en nuestro artículo explican algunas de las razones extra-textuales que Ilevaron a Labou Tansi a escribir L'anté-peuple. Los modelos latinoamericanos que tal autor hace suyos en sus demás novelas corresponden a los proporcionados por las obras de García Márquez. Pageaux (1982; 1985), Séwanou Dabla (1986), Ananissoh (1997), Abdoulaye Diop (2002) y Paravy (2011), con algunas variaciones entre sí, abordan la literatura del escritor co- lombiano y la del congoleño desde una perspectiva comparada.

Para llevar a cabo nuestro análisis, dividiremos las páginas que siguen en dos partes: la primera analiza la forma en la que L'anté-peuple reelabora la invariabilidad de condiciones político-sociales vividas en el Zaire y en la República Popular del Congo durante la época en la que fue escrita tal ficción; la segunda, en cambio, indaga sobre la manera en la que sus personajes sobresalen por encima de las circunstancias que deben enfrentar. A partir de ello, en las conclusiones, concebimos L'anté-peuple como una novela cuya estética es compleja debido a los elementos que configuran de modo particular el espacio, el tiempo y sus respectivos valores dentro de la ficción. Del mismo modo, consideramos algunos de los temas de la obra analizada que pueden ser abordados en estudios ulteriores y dejamos abiertas las perspectivas para ahondar en la literatura del escritor elegido y en su tradición literaria y cultural.

\section{INMUTABILIDAD DE LAS CONDICIONES POLÍTICO-SOCIALES}

El análisis que proponemos considera, en primera instancia, el espacio ficcional de L'anté-peuple. Su historia se desarrolla entre el Zaire (hoy, República Democrática del Congo) y la República Popular del Congo (hoy, República del Congo), a ambas orillas del río que los separa (Ngate, 1988; Thomas, 2002). Ahora bien, el mundo novelesco de la obra que nos ocupa es un lugar en el que ocurren grandes cambios. Dentro de estos, existe una cierta disolución de las costumbres causada en parte por la caída en desuso del fundamento cultural tradicional. Así, la atmósfera de mediocridad que impregna a Kinshasa, en el Zaire, y a Brazzaville en el Congo, muestra los efectos de un derrotismo mórbido. En ambas ciudades, el gusto por el lucro, la megalomanía y la sed de poder han suplantado el respeto por el otro y todo sentido de equidad. Aparece una Kinshasa cuyo pulso late al ritmo del regreso a la autenticidad promulgado por Mobutu mientras que, sobre la otra orilla, se esboza una Brazzaville que está cambiando al ritmo del "socialismo científico" representado por Marien N'Gouabi (Kapanga, 2001). Kinshasa y Brazzaville se presentan así como una suerte de hermanas gemelas 
cuyos destinos están ligados entre sí, de tal suerte que la menor agitación que ocurra en una se sentirá con intensidad redoblada en la otra.

Sobre este escenario — un mundo real ficcionalizado mediante el trabajo estético desarrollado por Labou Tansi-, el lector asiste al punto de partida sobre el que se teje la inmutabilidad que la obra propone. Las situaciones que se viven en ambas ciudades mantienen a sus habitantes sumidos en condiciones de vida perennes que atentan contra su dignidad y su integridad humana. Moverse de un lado del río que los separa al otro es, por ende, un desplazamiento geográfico sin mayores modificaciones en la forma de vivir de quienes migran.

Una mirada general a los dirigentes políticos encargados de gobernar cada uno de estos países nos permitirá adentrarnos en la idea de inmutabilidad que L'anté-peuple plantea. El 24 de noviembre de 1965, Joseph Désiré Mobutu ascendió al poder en el Zaire mediante un golpe de Estado; se mantuvo al frente del gobierno hasta el 17 de mayo de 1997, cuando Laurent Désiré Kabila ocupó su lugar. Mobutu debió exiliarse en Marruecos (Becerra y Piatti Martínez, 2005; Kabunda Badi, 2003; Mària Serrano, 2005). A partir de la lectura que hacen de Castells (2004), Shmite y Nin $(2007,2009)$ sostienen que Mobutu estructuró su poder sobre la base de la única sección operativa del ejército: la guardia personal. Al mismo tiempo, repartió los cargos políticos y militares entre las distintas etnias. De este modo, logró protegerlas y controlarlas mientras que, de forma simultánea, incentivaba confrontaciones violentas. Además, "pas[ó] gran parte del tiempo en sus mansiones de Suiza, Francia, España y Portugal" (Shmite y Nin, 2007, p. 133; Shmite y Nin, 2009, p. 76). El suyo fue un gobierno que implementó un régimen etnofascista, de depredación, fundamentado en el autoritarismo, la corrupción y en una represión sin precedentes, lo cual permite comprender la amplitud que tuvo su mandato en el tiempo. Así, su régimen se caracterizó por la impunidad y por el desvío de fondos públicos como modo de llevar adelante sus políticas (Kabunda Badi, 2000).

En el Congo, por su parte, Marien N'Gouabi tomó el poder en agosto de 1968, también mediante un golpe militar que derrocó a Alphonse MassembaDebat, un socialista moderado que había fundado años antes el Movimiento Nacional de la Revolución (MNR) como único partido. Pese a haberse reivindicado como un socialista científico y a haber tomado algunas medidas radicales, Massemba-Debat fue considerado más como un tecnócrata que como un ideólogo (Clark, 1994). N'Gouabi se convirtió en presidente de su país en enero de 1969 y se mantuvo en el cargo hasta su asesinato en marzo de 1977. Tras su muerte, Joachim Yhombi-Opango ocupó la presidencia hasta 1979 y, desde entonces, Denis SassouNguesso, quien se mantuvo en funciones hasta 1992 (Lee, 2006; Eaton, 2006). Ahora bien, el ascenso de N'Gouabi al poder supuso una reorientación fundamental en la política del Congo que perduró hasta mediados de 1991. N'Gouabi declaró al Congo un estado marxista-leninista, anunció la adopción de una nueva constitución, proclamó una bandera e himno nacional de corte comunista y creó el Partido Congoleño del Trabajo (PCT), el cual comprometió a los líderes del país con el desarrollo "marxista" durante dos décadas (Clark, 1994). Desde este momento y hasta la culminación del mandato de N'Gouabi, la retórica radical, los lazos con China y con la Unión Soviética y el rígido control económico establecido por el Estado fueron los ejes de la política gubernamental. No obstante, también se preservó la relación cercana del Congo con Francia (Eaton, 2006).

Las condiciones político-sociales generadas por Mobutu y por N'Gouabi a ambos lados del río Congo, junto con el correlato cultural inherente a ellas en los países que gobernaron, configuraron, a su manera, situaciones que se extendieron en el tiempo. La cultura, entendida "como el sistema significante a través del cual, necesariamente (aunque entre otros medios) un orden social se comunica, se reproduce, se experimenta y se investiga" (Williams, 1994, p. 13), se unió a la política para moldear circunstancias que perduraron por decenios. Se presenta, por un lado, el mandato de un único dirigente que se prolongó durante más de treinta años, quien ostentó el poder mediante el dominio de distintas esferas estatales y se lucró a costa de los recursos nacionales y de los fondos públicos del Estado. Por otro lado, se da una sucesión de cambios de mandatarios pertenecientes 
a un mismo sistema político cuya bifurcación dio origen a la creación de un partido con lineamientos que perduraron durante más de dos décadas. Tanto una como otra situación conllevaron a visibilizar la persistencia de una realidad áspera vivida en los nacientes países africanos. Sus habitantes, ubicados a lado y lado del río, padecieron las consecuencias sin fin de las administraciones estatales correspondientes. Sus vidas asistieron así al suplicio de ver, año tras año, cómo se repetían algunos comportamientos corruptos y ciertos lineamientos gubernamentales que atentaron contra las libertades personales y colectivas. De esta manera, la degradación del hombre por el aparataje político puesto en marcha en el interior de cada frontera se tornó en moneda corriente. Ese es el marco extra-textual en el que Sony Labou Tansi escribió L'anté-peuple.

Ahora bien, esos mismos regímenes y las consecuencias culturales que trajeron consigo son los que, paralelamente, se elaboran en L'anté-peuple. Mientras que en un primer momento se observa a Dadou compartiendo espacio con personalidades del gobierno, su incriminación y su encierro en prisión por la muerte de Yavelde lo ubican en las antípodas del círculo social al cual pertenece (Labou Tansi, 1983). Una vez ahí, es Nioka Musanar —el tío de la difunta- quien, desde el lugar que ocupa en el gobierno, toma el caso de su sobrina como un asunto personal a arreglar:

[Nioka Musanar] montait résolument les marches du pouvoir. De commissaire urbain, il était passé gouverneur communal, ministre de I'Information, puis actuellement conseiller à la présidence de la République. Oh ! s'il pouvait oublier. Mais non. Il se souvenait. Et s'il en avait décidé autrement, Dadou aurait été soulagé du petit acte de respirer. Mais il avait voulu qu'il restât à bouffer le béton.

Et quand on lui avait signifié que le régisseur ménageait son prisonnier, il était personnellement passé lui dire : «Cette ordure, c'est mon cas personnel. II faut lui compliquer le séjour» (Labou Tansi, 1983, p. 98, Comillas del autor).

El encarcelamiento de Dadou supone un control casi absoluto sobre él por parte del gobierno, pues la pri- sión "da un poder casi total sobre los detenidos; tiene sus mecanismos internos de represión -y de castigo: disciplina despótica" (Foucault, 2002, p. 238). El Estado, cuyo papel es desempeñado por Nioka Musanar en la parte de la novela ambientada en Kinshasa, encarna dos de los tres rasgos estadocráticos o de forma de gobierno con partido único que se implementaron en el África subsahariana. Por un lado, permite observar que hay un poder personal que transforma a los gobiernos de tal región en patrimonialistas y clientelistas. Quienes pertenecen al partido político quedan a disposición de la imagen del jefe, con lo cual se convierten en un séquito de funcionarios que toman parte en el otorgamiento de beneficios según el grado de cercanía que mantienen con el dirigente principal del país. Por otro lado, da cuenta de la proclividad a disminuir las libertades civiles y del empleo desmedido de la violencia como mecanismo para la resolución de conflictos que emergen de la relación entre gobernantes y gobernados. El tercer rasgo, ausente en la ficción que analizamos, es el nivel elevado de dependencia respecto a los Estados industrializados y de las entidades financieras internacionales (Shmite, 2009). Más adelante en la novela, el lector puede observar que las ansias de poder llevan a Musanar a atentar contra la vida del comisario general del pueblo - antiguamente Ilamado presidente de zona- (Labou Tansi, 1983).

La cárcel es el espacio a través del cual se revelan otras formas adoptadas por el régimen político instaurado en el país y por la cultura derivada de este. La detención de Dadou llevada a cabo en ella permite observar el manejo constante de la represión colonial que se antepone a la protección. Asimismo, da cuenta de las formas de poder personalizado que, para la época en la que se escribió el libro, eran recientes y excéntricas en el África. Lo anterior difiere del modelo de Estado weberiano o foucaultiano, fundamentado en métodos burocráticos de mando, control general e interiorización del rigor extendido por parte de los ciudadanos (Bernault, 2007). Aunque de forma concisa en la novela, en la prisión se observan tanto los abusos físicos como los demás avatares que los nuevos gobiernos, erigidos tras las independencias, trajeron consigo para quienes estuvieron vinculados 
con la administración pública y para aquellos que militaron por las causas políticas en el Zaire.

Los primeros —abusos físicos perpetrados contra los personajes principales de la novela- se pueden apreciar cuando el narrador alude a la situación de violencia sexual de la que Yealdara es víctima cuando es detenida junto a Dadou y el taxista que los transporta hasta el domicilio del protagonista (Labou Tansi, 1983). Pese a que el escritor dedica poco espacio a tal situación, consideramos que es un aspecto bajo el cual se observan las agresiones cometidas por los funcionarios del sector público en el régimen gubernamental del momento en el Zaire. Es Ilamativo que el autor dedique poco espacio a las agresiones sexuales experimentadas en la estación de policía. No obstante, creemos que tal situación se debe a que ellas son sufridas por Yealdara, quien adquirirá poco a poco una fuerza y un rol cruciales en la ficción hasta desempeñar un papel muy cercano al del protagonista. El desarrollo del personaje de Yealdara conlleva así a que se aluda a la violación que vive sin profundizar en ello, lo cual realza la fuerza con la que se sobrepone a las circunstancias de su tiempo. Volveremos sobre esta cuestión en el segundo apartado del artículo.

En cuanto a los segundos - las vicisitudes que tuvieron los regímenes nacientes para quienes trabajaron en la administración pública-, la cárcel permite observarlos a través de Falodiati. Con él, la ficción muestra a la clase social conformada por los funcionarios estatales y el destino al que estos estuvieron condenados. Sobre él, el narrador afirma que su encarcelamiento ha sido producto de una forma de actuar con consecuencias inevitables: "il avait assassiné deux millions de zaïres au préjudice de l'État [...C]'est dur d'être fusillé pour détournement des deniers publics" (Labou Tansi, 1983, p. 82).

Asimismo, el estilo de vida de tal personaje antes de su encierro, con su familia, las chicas de su barrio, el vino caliente de la embajada de Bélgica, las orquestas, los caballos y los fines de semana en Matadi (Labou Tansi, 1983), permite vislumbrar su pertenencia a la pequeña burguesía zaireña, la cual usó su poder político para consolidar su poderío económico. Esto permitió que dicha clase social empleara el poder estatal con fines más próximos a los intereses personales que a la realización de tareas productivas. Tal situación desembocó en la creación de un Estado "neopatrimonial" o "rapaz", que se distinguió por el desvío de fondos económicos con propósitos políticos, la difuminación de los límites que separan lo oficial de lo privado que trajo consigo la privatización de los bienes públicos, el miedo, la apropiación del poder por parte de una única persona, la mala gestión estatal junto con la opresión burocrática, el clima político cambiante, la corrupción instaurada en todas las instituciones y la supresión del derecho (Kabunda Badi, 1994). Más adelante en la novela, el lector observa que Falodiati escapa de la prisión dos años antes que Dadou (Labou Tansi, 1983).

Con respecto a los acontecimientos que trajeron consigo las formas de gobierno instauradas en el Zaire para quienes militaron por las causas defendidas por los distintos grupos políticos del joven país, es el alcaide de la cárcel quien permite observarlos. La entrevista que él mantiene con Dadou en su despacho deja ver que, como el personaje principal, también estuvo encerrado en la prisión. En las páginas que narran el encuentro se observa que, como máxima autoridad del penal, tiene interés en conocer al recluso y en conocer por la propia voz de este si ha sido en efecto el autor del crimen que lo ha conducido hasta donde se encuentra (Labou Tansi, 1983). En ellas, también se evidencia un interés del alcaide por entablar un diálogo más cercano con el preso que le permita al primero alcanzar cierto grado de comunión con el segundo. Así, el alcaide dice al prisionero:

Ici, c'est un peu chez moi. J'y suis venu en 'prison de régime': c'est plus sale. C'est plus vide. Ça vous gerbe dans le cerveau. Longtemps, très longtemps, vous devenez la proie de votre propre peau! La cellule où vous êtes, je l'ai occupée. Je sais comme elle mord. Quatre ans que j’y ai passé. Quand le nouveau régime est venu, ils m'ont prêté un bout d'extérieur. Le bout du dehors qu'on m'a accordé, je l'ai exploité à ma manière [...]. Onze ans que je suis là. Je regarde. Ils passent -des coupables, des innocents-, génies, faussaires, simples. Évidemment, dans un monde où seul le coupable a sa place, que voulez-vous 
qu'on foute des innocents ? (Labou Tansi, 1983, p. 91. Comillas del autor).

El alcaide deviene, junto con Dadou, en uno de los sujetos sobre los cuales se ejerce con mayor fuerza el poder gubernamental. No solo es alguien que se encuentra sometido a los designios del régimen, sino que, además, su trabajo como autoridad de la cárcel lo somete al cumplimento de un rol dentro del aparato represivo del Estado que, a diferencia de los ideológicos, actúa a través de la violencia (Althusser, 2003). Su discurso permite observar la conciencia que tiene acerca del funcionamiento de la maquinaria estatal imperante. Asimismo, deja ver cómo el preso se va convirtiendo en carcelero de su propio cuerpo gracias al efecto del encierro y al hecho de naturalizar la represión impuesta por el régimen. Como miembro del aparato represivo, sabe que este tiene falencias debido al emplazamiento errado de inocentes que promueve. $Y$, sin embargo, posee total lucidez respecto a los vínculos que lo unen a tal maquinaria estatal. El presidio a su cargo, en el cual también fue recluso, ha sido el dispositivo mediante el cual el gobierno de su país se ha manifestado ante él. La prisión, entendida como instrumento de administración de poder y de muerte, forma parte de los mecanismos generales que hacen posible la gubernamentalidad del régimen (Mbembe, 1990).

La situación que se presenta del otro lado de la frontera que supone el río Congo es, a grandes rasgos, la misma que viven los personajes en su país de origen. La novela traza así una simetría entre los países involucrados en la ficción que propone y sus condiciones político-sociales y culturales. Tal correspondencia es aún más notoria si se repara en la aparición de Rita cuando Dadou se encuentra ya del otro lado de la frontera. El narrador afirma que el protagonista de la historia se asombra del parentesco que observa entre ella y Yavelde. Del mismo modo, tanto esta última como aquella mantienen una relación cercana con el gobierno de su respectivo país: Yavelde, por ser sobrina de Nioka Musanar; Rita, por pertenecer al Partido (Labou Tansi, 1983). Ahora bien, mientras que la difuminación de los límites entre los problemas personales y el ejercicio de las labores políticas de los funcionarios queda puesta en evidencia en Kinshasa por las acciones que conllevan a que Dadou sea recluido de forma injusta en prisión, en la República Popular del Congo se observa cuando el jefe de los rebeldes cuenta la historia de cómo él y algunos miembros de su grupo llegaron a la clandestinidad:

Vous savez comment je suis venu au maquis? Un mec de là-bas enviait ma femme. II m'a fait passer pour un maquisard. On voulait m'arrêter. J'ai descendu cinq bérets et j'ai foutu le camp. Je ne pouvais plus reculer du moment que ma place, là-bas, je l'avais tuée. J'ai couru devant, toujours devant. Parce que derrière moi, c'était le néant. J'ai tué d'autres bérets sur mon passage [...]. Mais là-bas comme mes papiers étaient morts comme ma place était morte -, je suis resté ici, d'autres sont venus : Santiago et Fouty-Mak [...]. Santiago avait fui la ville parce qu'on I'accusait d'avoir assassiné le maire. Sous l'accusation, il y avait une vieille histoire de cul. Fouty-Mak, lui, avait quitté la ville pour des raisons pas trop loin des nôtres. Le groupe grandissait. Tous ceux qui perdaient leur place là-bas, pour une raison ou pour une autre, venaient ici (Labou Tansi, 1983, pp. 203-204).

De acuerdo con lo anterior, es el abuso de poder el que lleva al jefe de los rebeldes y a sus compañeros a la clandestinidad y la disidencia. Debido a los problemas personales en los que se ven inmersos, distintas situaciones de sus respectivas vidas privadas se mezclan con aspectos vinculados con la política del régimen. Aunque ya no se observa a través de la cárcel como en el caso de Dadou, la forma de actuar de quienes gobiernan al otro lado del río Congo revela el control ejercido sobre la vida de quienes habitan el país al que los personajes centrales de la novela han llegado exiliados.

Tal situación adquiere una relevancia mayor si se observa a la luz del clima social que imperó en el país como producto de las condiciones políticas de la época en que se escribió tal obra. Durante el periodo de monopartidismo que vivió el país entre 1963 y 1990, primero con el Movimiento Nacional de la Revolución y luego con el Partido Congoleño del Trabajo, la élite política encargada de dirigir las fuerzas armadas de la República Popular del Congo fue la única que ejerció en ella la violencia 
(Bazenguissa-Ganga, 1998a; Bazenguissa-Ganga, 1998b). Bajo el mandato liderado por el último de aquellos partidos, el monopartidismo retomó los rasgos represivos propios de un aparato político unitario, tales como los arrestos políticos, la represión de las libertades, la prohibición de la expresión, el delito de opinión, entre otros (Kitsimbou, 2006). Así, tal como afirma Sundberg, en la época de la existencia de un único partido, los ciudadanos carecieron de derechos. En cambio, fueron constantemente hostigados por las fuerzas públicas y por los grupos paramilitares. En el caso de la policía, particularmente, esta fue empleada para cumplir planes secretos por parte de la élite política y no para proteger la vida ni la propiedad privada de los ciudadanos del común (1999).

El espacio, desplegado en su totalidad, adquiere del lado de la República Popular del Congo unas características particulares. Por ende, la prisión que en Kinshasa aparecía para recluir a Dadou como forma de ejercer poder sobre él adquiere con su ausencia otro significado, pues es la misma sociedad — configurada por las disposiciones políticas del régimen - la que se transforma en una suerte de cárcel. En ese sentido, ya no es un dispositivo de sometimiento cerrado el que mantiene cautivo al hombre, sino el contexto en sí el que le impide ser libre, con lo cual el ambiente sociopolítico y cultural - junto con todo lo que hay en él—configura una prisión a cielo abierto que oprime a quienes habitan de ese lado del río Congo. En esta línea, González Calleja (2006) entiende la represión como una acción que vulnera la integridad personal. Son los gobiernos, las organizaciones internacionales o algunos grupos profesionales los encargados de llevarla a cabo. En ese sentido, son organismos que poseen tanto la autoridad legítima o de facto como los métodos de coerción para lograr que se respete tal autoridad en tanto pieza dentro de un marco legal vigente. Así, la represión comprendida como el grupo de dispositivos "dirigidos al control y la sanción de conductas 'desviadas' en el orden ideológico, político, social o moral, aparece como un concepto muy cercano a la noción de violencia política" (González Calleja, 2006, p. 554. Comillas del autor). Es esa represión, ejercida sobre los cuer- pos y también sobre la ideología y la moral, la que configura la cárcel sin rejas a la que ingresa Dadou.

Al respecto, abordar el pueblo de los pescadores nos permitirá ilustrar la forma en que se configura la prisión a cielo abierto mediante la represión que se ejerce $y$, del mismo modo, la manera en la que el régimen de la República Popular del Congo se hace presente. En tal aldea, el régimen se observa mediante dos formas. La primera de ellas es la del abuso físico que implica la desaparición forzada de personas por parte de los militantes del partido. En su trabajo sobre la represión, González Calleja (2006) expone los cuatro niveles que esta adquiere en la teoría de Michael Mann. La escalada de la represión militar es el último de ellos. Tal nivel de opresión es el que se observa en la novela de Labou Tansi. Desde la llegada de Dadou al otro lado del río, las detenciones políticas aparecen de forma recurrente. Fortuné Loupanzo, conocido como Sacramento, los hijos gemelos de la anciana que recibe al protagonista en el pueblo de los pescadores e incluso el protagonista y las personas que lo cuidan son víctimas de arrestos por parte del ejército. Más adelante, otros personajes tales como el alcaide — quien también llega en condición de exiliado al país - y Amando, el anciano que brinda resguardo a Yealdara, también son privados de su libertad por el Estado (Labou Tansi, 1983).

La segunda forma es la del aislamiento. Retomar los planteamientos de Foucault contribuirá a desarrollar nuestro argumento. Para él, el alma moderna constituye un elemento generado a partir del poder remanente infligido sobre los cuerpos sometidos, con una existencia y una realidad que la produce constantemente alrededor, en el exterior y también dentro del cuerpo debido al funcionamiento de un poder ejercido tanto contra quienes se castiga como contra aquellos destinados a ser supervisados, educados o corregidos —es decir, aquellos a los que se vincula a un mecanismo de producción para ser controlados durante toda su vida- Tal alma, surgida de los métodos de castigo, vigilancia, pena y coacción, organiza los resultados de cierta clase de poder y la información referida a un saber: la estructura mediante la cual los nexos del saber permiten un saber posible, a la vez que este extiende y asegura los resultados del poder. A todo hombre, dentro de una sociedad 
específica, "Un 'alma' lo habita y lo conduce a la existencia, que es una pieza en el dominio que el poder ejerce sobre el cuerpo. El alma, efecto e instrumento de una anatomía política; el alma, prisión del cuerpo" (Foucault, 2002, p. 36. Comillas del autor).

Es esa alma moderna, entendida como el producto de la sociedad de la vigilancia y el control que acompaña al sometimiento físico, la que complementa el cautiverio experimentado por los personajes en la República Popular del Congo. Con ella, el régimen afianza su poderío. La conversación que mantiene la anciana que alberga a Dadou con él a propósito de la detención de Fortuné Loupanzo (Sacramento) y la situación política del momento, junto con las observaciones que Henri — uno de los nietos de la anciana—, hace al protagonista acerca del lugar que debe ocupar en tanto pescador dentro de la sociedad instaurada por el régimen, permiten evidenciar tal situación:

» Ici on ne parle politique qu'à la réunion. Après, ça s'appelle rébellion et c'est sévèrement puni.

-C'est la première fois que je parle de ces choses, dit Dadou.

-Ici on n'en parle pas, insista la vieille.

-C'est partout comme ça, dit Dadou. Partout.

-Nous, ici, on parle du fleuve et des poissons. Après le fleuve, après les poissons, c'est la prison (Labou Tansi, 1983, p. 134).

[...]

-Qu'est-ce qu'un pêcheur a à voir avec la cité du Parti ? C'est là des choses qui ne nous regardent pas. On a construit une vingtaine de cases extraordinaires à deux kilomètres d'ici : c'est gardé par les bérets. Vous, un pêcheur, qu'est-ce que vous iriez faire là-bas (Labou Tansi, 1983, p. 140).

El diálogo entre la anciana y Dadou y las palabras que este recibe de Henri revelan tanto la carencia de libertad de expresión como el aislamiento de ciertos ámbitos sociales que experimentan los pescadores. Los habitantes del pueblo solo están habilitados a hablar de los temas ajenos a la organización estatal y a la política imperante. Asimismo, se encuentran apartados de aquellos espacios en los que tienen lugar los asuntos del país, lo cual demuestra el sometimiento que viven respecto a sus gobernantes. Así, padecen un confinamiento que se transforma en dominación por parte del régimen y en reclusión autoimpuesta.

De acuerdo con Lassi (2012), aunque el pueblo de los pescadores posee medios de autosuficiencia alimentaria, es la frustración derivada de la resignación a ser ciudadanos de segunda clase que experimentan sus habitantes lo que define a la aldea. En la ficción, son considerados menos que hombres. Se menosprecian y se autoexcluyen, pues han interiorizado el menosprecio que han observado en la apreciación de sus vecinos del partido, quienes viven en el casco urbano. La desestima que experimentan de sí mismos es una consecuencia de la represión con que las autoridades han demostrado el poco valor que para ellas tiene la vida de los habitantes del pueblo cuando estos han intentado obrar como seres humanos, brindando opiniones sobre política, reclamando derechos o protegiendo presuntos miembros de la oposición al régimen. Dentro de los apartados de la novela en los que este investigador se basa para formular tales aserciones se encuentran los que nosotros hemos empleado en la cita que precede a este párrafo. Al retomarlos de forma literal, concordamos con sus planteamientos referidos a la marginalización ecológica, el debilitamiento de la identidad, la discriminación ambiental y la violencia experimentada por algunos personajes de Labou Tansi. No obstante, por nuestro corpus de análisis y a diferencia de Lassi, únicamente lo hacemos para el caso de la novela que nos ocupa.

Los acontecimientos narrados en L'ante-peuple, ambientados a lado y lado del río Congo bajo el mandato de Mobutu en el Zaire y con el gobierno de N'gouabi en la República Popular del Congo, muestran una constante en la represión y en el abuso de poder por parte de sus dirigentes y de los funcionarios estatales que trabajan para ellos. Los regímenes instaurados en ambos países se hacen sentir en distintos planos de la vida de sus respectivos habitantes. Ambos Estados adquirieron una condición casi similar, lo cual refleja las trayectorias históricas compartidas. El río que los designa y que al mismo tiempo demarca sus límites da cuenta de ello en múltiples planos. En la ficción, debido a la confusión intencional de 
ambientes que se origina por los sucesos narrados, lo Otro se transforma en lo Mismo. Cualquier crítica formulada a uno se vuelve aplicable al otro de manera simultánea. De esta forma, cada uno de los dos Estados implicados se vuelve una versión del otro (Ngate, 1988; Thomas, 2002). A esta condición de inmutabilidad se antepone el cambio experimentado por los personajes.

\section{TRANSFORMACIÓN DE LOS PERSONAJES}

Si el espacio de L'anté-peuple se muestra como invariable a ambos lados del río Congo, los personajes principales de la novela aparecen, en cambio, como entidades ficcionales que mutan a lo largo de la historia. Las condiciones vividas por ellos en ambos países los llevan a experimentar transformaciones en sus acciones que contrastan con el entorno que los rodea, lo cual complementa el significado global de la obra. Mientras que el ambiente en el que se mueven - hostil, colmado de privaciones y de abusosaparece ante los ojos del lector como inalterable, los caracteres centrales propuestos por Labou Tansi y sus formas de desenvolverse en su mundo evolucionan constantemente para hacer frente al contexto político y sociocultural en el que habitan. En ese sentido, son ellos y no el medio circundante quienes ocupan el primer plano de la ficción, con lo cual la interpretación que de esta hacen los lectores toma un significado de resistencia especial. L'anté-peuple trasciende, así, los límites de una literatura ligada a la simple elaboración literaria de los conflictos regionales propios de su tiempo y espacio de enunciación para constituirse en una obra que se contrapone a las circunstancias en medio de las cuales surge. Son las acciones de los protagonistas, entendidas como "las grandes articulaciones de la praxis (desear, comunicar, luchar)" (Barthes, 1993, p. 187. Cursivas del autor), las que los ponen por encima de su entorno.

En este punto, el trabajo de Kapanga (2001) adquiere una relevancia crucial para nuestro estudio. Para él, el argumento de la novela que analizamos permite observar una evolución tripartita que se concreta con el personaje de Dadou: "une étude minitieuse révèle que la trame du roman reflète une évolution ternaire comprenant une prise de conscience de l'injustice régnante, la recherche rationnelle d'une solution adéquate, et enfin un engagement radical pour le changement" (p. 184). Visto a la luz de la teoría de Barthes, el estudio de Kapanga plantea así una secuencia. Esta es "una sucesión lógica de nudos, unidos entre sí por una relación de solidaridad: la secuencia se abre cuando uno de sus términos no tiene antecedente solidario, y se cierra cuando otro de sus términos no tiene ya consecuente" (Barthes, 1993, p. 182). El trabajo de Kapanga se ocupa de organizar la forma en la que se desenvuelve el personaje principal de la novela. En este sentido, analiza cada uno de los tres estados por los que Dadou debe atravesar, lo cual implica la última secuencia de funciones que permiten alcanzar la acción general llevada a cabo por el personaje. Ahora bien, si se observa la serie que esos tres momentos componen a la luz de la teoría formulada por Barthes (1993) para el análisis estructural de los relatos, tal acción es la de actuar: obrar como forma de enfrentar las condiciones que el tiempo y el espacio imponen al hombre.

Al obrar, Dadou toma distancia de sí mismo, con lo cual se comporta de una forma distinta; tanto de su manera de ser como de la de aquellos que lo rodearon hasta el momento en que emprende su obrar al final de la novela. Se aleja de su actitud pasiva frente al mundo y también de la postura de resignación e indiferencia que sus semejantes toman frente a las condiciones sociopolíticas y culturales que oprimen a los habitantes de cada uno de los países que ha tenido la oportunidad de habitar. Pese a que alberga dudas en su interior, el Dadou que se ve forzado a luchar contra un mundo que enajena al hombre difiere mucho de aquel que, encerrado en prisión, reflexionaba sobre las condiciones individuales de existencia del ser humano y desdeñaba a los demás (Kapanga, 2001). En este sentido, tener la habilidad de actuar de una manera diferente implica poseer la capacidad de participar en el mundo, o de inhibirse de tal participación, "con la consecuencia de influir sobre un proceso o un estado de cosas específicos [...]. Una acción nace de la aptitud del individuo para 'producir una diferencia' en un estado de cosas o curso de sucesos preexistentes" (Giddens, 2006, p. 51. Comillas del autor).

El capítulo final de la novela es fundamental para comprender la trasformación de sus personajes 
principales. Además de ser el que cierra la última secuencia de funciones y, por ende, alcanzar el nivel de la acción que Dadou lleva a cabo en la historia, es el que también permite observar los matices que adopta tal acción. Esta se desdobla en dos formas. La primera de ellas es la de la participación activa: en el campamento de rebeldes que acoge al personaje principal, el jefe solicita voluntarios para llevar a cabo una misión; Dadou se ofrece para realizarla, pese a que el narrador de la historia relata la poca estima con que sus compañeros de campamento observan las cualidades militares del personaje principal. Aunque el jefe brinda la oportunidad de que su subordinado piense con detenimiento las cosas, este último se obstina en ser él quien ejecute el objetivo militar que le ha sido encomendado: matar al Primer Secretario. Después de cierto tiempo de espera, logra su cometido (Labou Tansi, 1983).

La segunda forma que adquiere la acción es, paradójicamente, la de la inhibición: luego de que Dadou ejecuta su objetivo, se dirige a casa de Assabrou, un amigo de Amando; poco después llega también Yealdara. La conversación que mantienen en conjunto mientras el protagonista come permite observar la abstención de Dadou de participar de discursos relacionados con doctrinas políticas:

-Mange, dit Assabrou. Notre cause est juste : parce que, si la vie cesse d'être sacrée, la matière, toute la matière ne sera plus qu'une sourde folie.

-Je n'ai pas besoin d'idéologie, dit Dadou. Les choses se bousculent si vite dans ma tête et je me demande si je ne suis pas déjà fou pour de vrai. Vous ne pouvez pas comprendre : j'ai tué parce qu'on m'a dit : va tuer. Après, c'est la nausée. L'odeur du sang. Ah! ma mère!

- C'est la guerre, dit Assabrou. Ceux qui meurent ont plus de chance que ceux-là qui les tuent. Mais parlons d'autre chose, voulez vouz?

-J'ai sommeil, dit Yealdara (Labou Tansi, 1983, p. 210).

La intención de evadir discursos políticos por parte de Dadou, sumada a la intervención final de Yealdara, permite al lector pensar en la inhibición como una manera distinta de ejercer la acción. Esta afir- mación adquiere mayor fuerza si se observa a la luz de las acepciones que pueden atribuirse al sustantivo sommeil: por un lado, expresa el deseo de dormir; por otro - bajo un registro lingüístico formal-, expresa la inacción o inactividad. La expresión francesa correspondiente a la segunda acepción es en realidad un sintagma preposicional: en sommeil (en reposo, en inactividad o en inacción). Sin embargo, desde la óptica bajo la cual analizamos la novela, consideramos que Labou Tansi se aventura a jugar y a romper con ciertos mecanismos sintácticos de la lengua francesa para proponer un significado propio mediante sus ficciones. Tal procedimiento es un ejemplo de cómo el autor hace suyo el francés, lengua de uno de los colonizadores del territorio africano. Con estos planteamientos, nos aproximamos a los de Kapanga (2001) sobre el mismo asunto. En este sentido, aunque Dadou y Yealdara han obrado de forma activa para hacer frente a los mecanismos de opresión instaurados por los gobiernos de Mobutu y Marien N'Gouabi, optan por eludir las palabras de su anfitrión acerca de la causa política que conlleva a los rebeldes a mantener su lucha.

De esa manera, tanto Dadou como Yealdara ejercen su agencia, entendida como "la capacidad socialmente determinada para actuar y establecer una diferencia" (Barker, 2003, p. 270). Cuando elige asesinar al Primer Secretario, Dadou se encuentra dentro de un grupo de rebeldes refugiados en la selva; Yealdara, en cambio, decide formar parte del grupo de locos integrados a la organización opuesta al sistema como forma de encontrar a Dadou, lo cual la lleva a luchar al lado de ellos. En eso radica su forma de actuar para marcar una diferencia, pues el contexto sociopolítico los impulsa a la clandestinidad. Sin embargo, con el atentado al Primer Secretario y las represalias, las condiciones impuestas por el contexto varían y la determinación social de su aptitud para obrar sufre un giro completo: ya no se trata de adherir a una agrupación para plantear la divergencia; esta se formula mediante la pasividad que evita la intervención en asuntos subversivos cuyos resultados podrían atentar contra la vida de los hombres del común.

Si la acción que Dadou lleva a cabo es la de obrar, el rol reservado para Yealdara es el de posibilitar la metamorfosis de aquel personaje hasta que pueda ejercer 
su capacidad de actuar. El cambio en el pensamiento del personaje principal de la novela no puede acontecer sino gracias al enfoque racional propiciado por la ayuda que ella le brinda. Así, para salvarlo de su inactividad, la historia la presenta como un agente de transformación. Ella es la encargada de iluminar el camino del protagonista (Kapanga, 2001). Yealdara alumbra el sendero interior que Dadou debe seguir, lo cual la lleva a atravesar todas las pruebas que se interponen en su trayecto hasta llegar al otro lado de la frontera (Makita, 1997). Una vez estando del otro lado del río Congo, incluso, continúa afrontando desafíos para alcanzar su objetivo. De ahí que, luego de resistirse durante mucho tiempo, acepte verse con Marti Mouyabas — el Primer Secretario del Partidoy emplear sus encantos femeninos contra él como un mecanismo de lucha: primero, para obtener los documentos legales que le permiten movilizarse por la ciudad; luego, para hacer algo con la desaparición de sus amigos. Asimismo, es lo que la lleva a aceptar pasar por el rito de iniciación al que se somete para ingresar al grupo de locos disidentes (LabouTansi, 1983). Así, se transforma y al mismo tiempo posibilita el cambio.

Como personaje encargado de contribuir a los procesos de transformación en la novela, Yealdara no solo coadyuva al cambio que opera sobre Dadou, sino que además permite que se lleve a cabo una variación respecto al papel que desempeñan los personajes femeninos que entran en relación íntima con el protagonista de la historia. Observar tal variación con base en los planteamientos de Simone de Beauvoir contribuirá a apreciar su importancia. En El segundo sexo, tal filósofa afirma que la mujer no solo se concibe como la esencia de casas, familias u hogares, sino también de grupos humanos más amplios, tales como: ciudades, provincias o naciones (de Beauvoir, 2014). La relevancia de esta perspectiva radica en las posibilidades que ofrece para pensar en cada una de las mujeres vinculadas a Dadou y su nexo con el tipo de nación al que pueden relacionarse: mientras que Yavelde se encuentra más próxima al Estado poscolonial que, infestado de corrupción política, expulsa a Dadou, Lola, la esposa de este, se halla más cercana al estadio previo bajo el dominio europeo, dado a que está presta a la seducción y a la posesión por parte de los colonizadores. La carta leída por el protagonista mientras se desplaza a través del río sustenta nuestra interpretación: "tu avais laissé tout mon corps me monter à la gorge. Et j'ai crié ; c'est beau quand une femme crie. Je suis allée avec le Belge. Pour que mon cri me semble plus sale" (Labou Tansi, 1983, p. 129).

Yealdara, en cambio, representa el ideal de nación independiente del dominio europeo. Al comienzo de la novela y mientras baila con Dadou, es ella misma quien revela su formación como doctoranda en Sociología en la Universidad Nacional del Zaire; es ella misma quien afirma haber necesitado un hombre inteligente, con sangre e ideas surgidas desde el fondo de sus entrañas (Labou Tansi, 1983). Así, Yealdara posee una educación con impronta humanística y social que le permite reflexionar sobre su mundo e interactuar con la comunidad en la que se encuentra inmersa. De igual modo, demanda para sí un individuo idóneo, dueño de su propio pensamiento y con energía vital emanada en el interior de su ser que le permita ser digno de ella y construir un entorno más allá de las fronteras remanentes que quedaron tras las independencias. Aunque la relación que entabla con Dadou es la de un amor no correspondido, la atracción que ella siente por él permite pensar en los anhelos de cierto ideal de hombre por parte de la nación deseada. Con ello, la ficción atiende a la interpelación de su contexto de enunciación relacionada con los problemas estructurales padecidos a ambos lados del río Congo.

Al respecto, Lassi (2012) afirma que el descolonizado vive inmerso en el caos. De acuerdo con este investigador, volver a hacer suyo su universo cultural y su ambiente geográfico es un asunto problemático para aquel que ha roto las cadenas del dominio ejercido por otras naciones. En aras de que aquellos que habitan su entorno e incluso ella misma tengan la posibilidad de volver a poseer su espacio, Yealdara es capaz aun de dejar atrás su vida como hija de Nioka Musanar en Kinshasa y de emplear sus encantos para luchar contra los miembros del régimen político instaurado en el país que la recibe:

Au village, une voisine vint lui annoncer qu'on avait emmené le vieux. 
-Je me vendrai pour lui, dit-elle à voix morte. Je me donnerai (Labou Tansi, 1983, p. 175).

Elle se remit à chercher l'adresse de l'attaché. Le jeune homme avait le sourire et l'oeil assez dupes. Elle pourrait lui tirer des choses sans se laisser cracher dans les cuisses. Ou, si les moyens refusaient d'obéir à cette hauteur des choses, elle se laisserait... Après elle irait se frotter de tous les savons du monde. Et ça partirait. La conscience. Elle n'avait qu'à obéir aux réalités, la conscience [...].

Yealdara lut l'adresse à haute voix.

» Marti Mouyabas... (Labou Tansi, 1983, p. 178).

Yealdara entrega así su cuerpo a la causa política por la que está luchando. A diferencia de Yavelde y de Lola, sus actos sexuales se encuentran ligados a la resistencia y a la subversión. Utiliza sus atributos cuando el régimen se lleva a sus amigos, lo que permite pensar, así, en un empleo razonado de su belleza al que recurre cuando la situación que vive ella y los que la rodean alcanza su punto máximo en la escala de opresión. Con sus encantos, espera convencer al Primer Secretario para que la ayude. Aunque en un principio tan solo piensa en un uso de su hermosura sin mayores implicaciones carnales, está dispuesta a hacerlo todo para obtener lo que necesita sin experimentar por ello sentimientos de culpa y de pudor, pues sabe que la conciencia responde a realidades determinadas según tiempos y espacios específicos.

De acuerdo con lo anterior, concordamos con Etoke (2006) cuando afirma que el cuerpo femenino es, por un lado, arma sexual; por otro, catalizador de cambio político. Aunque tal afirmación se basa en La Vie et demie, consideramos que aplica también para L'anté-peuple si se toma en cuenta que Yealdara prefigura a Chaïdana (Chemain, 1988), personaje de la primera de las novelas mencionadas. No obstante, el trabajo de Etoke también ve en los cuerpos femeninos concebidos por Labou Tansi la configuración de un conjunto de estereotipos que determinan a las mujeres de acuerdo con su sexualidad, lo cual elimina — desde la perspectiva de la investigadoracualquier tentativa de subjetividad del segundo sexo. Esto la lleva a formular una crítica vehemente al escritor que nos ocupa y a rechazar la manera en que este elabora, en su ficción más conocida, a la mujer. Ahora bien, desde la óptica bajo la cual elaboramos nuestro análisis, la carne deviene en Yealdara instrumento que tiende a alcanzar un fin dentro de un conflicto, con lo cual suprime la satisfacción del deseo y del placer para encaminarse — con todo su serpor las vías de la confrontación al sistema en apoyo a quienes se han opuesto al régimen imperante y a sus normas. Así, como mujer, se inserta en la lucha desde otro lugar distinto al que ocupan los hombres en la contienda física; aun cuando ello suponga que, ante el adversario, "su cuerpo ofrezca las cualidades inertes y pasivas de un objeto" (de Beauvoir, 2014, p. 159).

En un entorno inmutable liderado por gobiernos que oprimen a sus propios ciudadanos, Dadou y Yealdara cambian para oponerse así a las condiciones adversas en las que deben vivir tanto ellos como sus semejantes. En el caso del primero, la evolución que experimenta hasta alcanzar el nivel de la acción le permite adquirir la capacidad de obrar y de decidir sobre los acontecimientos que tienen lugar en el medio que lo rodea; en el caso de la segunda, es ella quien habilita la transformación del personaje principal. Además, en ella confluye la modificación que sufren los caracteres femeninos cercanos al protagonista, lo cual le permite estar más cerca de la línea en la que se lleva a cabo la lucha política. Juntos conllevan a pensar en el obrar y en el libre albedrío para hacerlo como formas de superar las dificultades que se presentan en los países liderados por Mobutu y por N'Gouabi. De este modo, se contraponen a las circunstancias desfavorables en las que se encuentran inmersos tanto su lugar de origen como el de llegada.

\section{CONCLUSIONES}

Nuestro trabajo ha expuesto la forma en la que L'anté-peuple elabora, mediante la literatura, las condiciones sociopolíticas y culturales en las que han vivido el Zaire y la República Popular del Congo bajo el mandato de Mobutu y de Marien N'Gouabi tras las independencias del dominio europeo. De forma análoga, plantea en Dadou y en Yealdara unos personajes que sobresalen entre los sucesos narrados como propuesta alternativa a los acontecimientos ocurridos en el mundo intratextual al que ellos 
pertenecen. Con ello, se configura un ambiente que logra su plenitud a medida que el lector va avanzando en su trabajo de interpretación. La novela que hemos analizado se erige así como una obra cuya estética es compleja en tanto ostenta una dialéctica en la que dos elementos antitéticos coexisten en relativa armonía dentro de la ficción, imbricándose de manera constante a lo largo de todo el libro: la inmutabilidad y el cambio. En tal complejidad radica su valor, pues ella permite que gracias al ejercicio de creación literaria se elaboren algunos de los aspectos históricos de los países en los cuales se desarrolla el argumento de la obra. Paralelamente, también posibilita que en esta última se conformen caracteres que con su actuar abren la perspectiva para pensar, desde la irrealidad de la ficción, en maneras de luchar contra las condiciones adversas vividas tras la instauración de los gobiernos poscoloniales. Con ello se establece una forma particular de cronotopo (Bajtin,1989): el de la acción —activa o pasivapara la transformación del entorno.

No obstante, aún hay que profundizar en el estudio de otros aspectos inherentes a la novela, tales como el papel semántico que posee la bebida dentro de la obra, el manejo que Labou Tansi lleva a cabo con la lengua francesa, y en los posibles nexos que L'antépeuple permite establecer con producciones literarias surgidas en América Latina debido a la temática tratada. Esto nos permitiría adentrarnos aún más en la tradición literaria y cultural del África subsahariana de expresión francesa, la cual aún sigue siéndonos ajena. De este modo, podríamos reconocernos en ella, pese a que tal ejercicio tan solo nos permita vernos reflejados como en siluetas todavía difusas. Así, iríamos más allá de los lindes nacionales en los que habitualmente permanecemos al adelantar nuestras investigaciones literarias para conocer qué de nosotros hay en los Otros situados al otro lado del Atlántico y cuánto de ellos, lejanos y en apariencia tan diferentes por su piel y sus tradiciones culturales, también nos habita.

\section{REFERENCIAS BIBLIOGRÁFICAS}

Abdoulaye Diop, E- H. (2002). Le réalisme social et magique chez Gabriel García Márquez et Sony
Labou Tansi. Éthiopiques, (68). Recuperado de http://ethiopiques.refer.sn/spip.php?article291

Adichie, C. N. (2009, julio). The danger of a single story [Archivo de video]. Recuperado de https:// www.ted.com/talks/chimamanda ngozi_adichie the danger of a single story \#t-12389

Althusser, L. (2003). Ideología y aparatos ideológicos del Estado. Freud y Lacan. Buenos Aires, Argentina: Nueva Visión.

Ananissoh, T. (1997). À propos de Sony Labou Tansi lecteur de García Márquez. Palabres: Revue culturelle africaine, 1(3-4), 59-98. Recuperado de http://www.revuepalabres.org/pdf/palabresplagiat.pdf

Bajtin, M. (1989). Teoría y estética de la novela. Madrid, España: Taurus.

Barker, C. (2003). Televisión, globalización e identidades culturales. Barcelona, España: Paidós Ibérica.

Barthes, R. (1993). La aventura semiológica. Barcelona, España: Paidós Ibérica.

Bazenguissa-Ganga, R. (1998a). Les milices urbaines au Congo: La transition démocratique et la dissémination des pratiques de violence. African Journal of Political Science / Revue Africaine de Science Politique, 3(1), 52-69.

Bazenguissa-Ganga, R. (1998b). Processus de démocratisation et élites politiques au Congo. Questions sur la violence urbaine. En G. Koubi et al., Questions sensibles (pp. 376-386). París, Francia: Presses Universitaires de France.

Becerra, M. J. \& Piatti Martínez, C. (2005). La guerra en la RDC, ¿Problema africano o condición para el desarrollo capitalista? Contra / relatos desde el sur, (1), 11-26.

Bernault, F. (2007). The shadow of rule: colonial power and modern punishment in Africa. En: F. Dikötter e I. Brown (Eds.), Cultures of Confinement: A History of the Prison in Africa, Asia, and Latin America (pp. 55-94). Londres, Inglaterra: Christopher Hurst.

Castells, M. (2004). La era de la información. Economía, sociedad y cultura. Vol. III. México DF, México: Editorial Siglo XXI. 
Chemain, A. (1988). Sony Labou Tansi, affabulation, critique sociale et ressourcement. Notre Librairie, (92-93), 132-133. Recuperado de https://gallica. bnf.fr/ark:/12148/bpt6k6503196k/f134.image

Clark, J. F. (1994). Elections, Leadership and Democracy in Congo. Africa Today, 41(3), 41-60.

de Beauvoir, S. (2014). El segundo sexo. Buenos Aires, Argentina: Debolsillo.

Devesa, J. M. (1996). Sony Labou Tansi: écrivain de la honte et des rives magiques du Kongo. París, Francia: L'Harmatann.

Eaton, D. (2006). Diagnosing the Crisis in the Republic of Congo. Africa: Journal of the International African Institute, 76(1), 44-69.

Etoke, N. (2006). Écriture du corps féminin dans la littérature de l'Afrique francophone: taxonomie, enjeux et défis. CODESRIA Bulletin, (3-4), 43-47.

Foucault, M. (2002). Vigilar y castigar. Nacimiento de la prisión. Buenos Aires, Argentina: Siglo XXI.

Giddens, A. (2006). La constitución de la sociedad. Bases para la teoría de la estructuración. Buenos Aires, Argentina: Amorrortu.

González alleja, E. (2006). Sobre el concepto de represión. Hispania Nova. Revista de Historia Contemporánea, (6), 551-579. Recuperado de https://www.redalyc.org/pdf/915/91500622.pdf

Kabunda Badi, M. (1994). La crisis del Estado y del desarrollo en África. Africa América Latina, cuadernos: Revista de análisis sur-norte para una cooperación solidaria, (14), 7-20.

Kabunda Badi, M. (2000). Impunidad, guerras y legitimidad democrática en África. Pasajes: Revista de pensamiento contemporáneo, (3), 15-29. Recuperado de http://roderic.uv.es/hand$\underline{\text { le/10550/45619 }}$

Kabunda Badi, M. (2003). Dimensión política y cultural de la conflictividad en la República Democrática del Congo. Nova África. (13), 7-26.

Kapanga, K. M. (2001). Esquisse d'un nouveau revolté social: le cas de Dadou dans L'Anté-peuple de Sony Labou Tansi. En: S. Z. Andrade, E. Julien, M. Rice-Maximin y A. Songolo (Eds.), Atlantic Cross-
Currents: Transatlantiques. Annual Selected Papers of the African Literature Association. Vol 9 (pp. 183-193). Trenton, Estados Unidos: Africa World Press.

Kitsimbou, X. B. (2006). La démocratie et les réalités ethniques au Congo (Tesis doctoral). Recuperado de https://tel.archives-ouvertes.fr/tel-00168467/ document

Labou Tansi, S. (1979). La Vie et demie. París, Francia: Éditions du Seuil.

Labou Tansi, S. (1983). L'anté-peuple. París, Francia: Éditions du Seuil.

Lassi, É. M. (2012). De la decolonisation a l'endo-colonisation: territorialite, environnement et violence postcoloniale dans les romans de Sony Labou Tansi. French Forum, 37(3), 163-180.

Lee, C. J. (2006). Republic of the Congo. En: N. Schlager, J. Weisblatt y O. J. Pérez (Eds.), World Encyclopedia of Political Systems and Parties (pp. 300-304). Nueva York, Estados Unidos: Facts On File.

Makita, P. (1997). L'anté-peuple ou l'exorcisme 'De I'amour et autres démons'. En: M. Kadima-Nzuji, A. Kouvouama y P. Kibangou (Eds.), Sony Labou Tansi ou la quête permanente du sens (pp. 329341). París, Francia: L'Harmatann.

Mària Serrano, J. F. (2005). De la guerra a la democracia: la República Democrática del Congo. Revista de fomento social, 60(238), 283-312.

Mbembe, A. (1990). Pouvoir, violence et accumulation. Politique africaine, 39, 7-24.

Ngate, J. (1988). Francophone African Fiction: Reading a Literary Tradition. Trenton, Estados Unidos: Africa World Press.

Pageaux, D.H. (1982). Gabriel García Márquez en français. De la traduction au modèle. Lendemains, (27), 45-52.

Pageaux, D.H. (1985). Entre le renouveau et la modernité: vers de nouveaux modèles ? Notre $L i-$ brairie, (78), 31-35.

Paravy, F. (2011). Écrivains africains en quête d'un tiers monde. En: C. Dumoulié y J. M. Moura (Orgs.), 
Entre la inmutabilidad y el cambio: una lectura de L'anté-peuple de Sony Labou Tansi

Actas del coloquio Les lettres francophones, hispanophones, lusophones et la latinité. Paris, Francia: Universidad de Paris Ouest-Nanterre-La Défense. Recuperado de http://www.revue-silene. com/images/30/article_87.pdf

Séwanou Dabla, J. J. (1986). Nouvelles écritures africaines. Romanciers de la seconde génération. Paris, Francia: L' Harmatann.

Shmite, S. M. \& Nin, M. C. (2007). África: un territorio de conflictos. Párrafos Geográficos, 6(1), 117147. Recuperado de http://igeopat.org/parrafosgeograficos/images/RevistasPG/2007 V6 1/7-6. pdf

Shmite, S. M. \& Nin, M. C. (2009). África: un territorio de conflictos. En: S. M. Shmite y M. C. Nin
(Eds.), Temas actuales, conflictos y fragmentación espacial ¿Cómo abordarlos desde la Geografía? Parte II. África como espacio geográfico de análisis (pp. 59-94). Santa Rosa, Argentina: Editorial de la Universidad Nacional de La Pampa.

Sundberg, A. (1999). The Problem of Change and Reorganisation of The One-Party Dictatorship in Congo. African Journal of Political Science / Revue Africaine de Science Politique, 4(2), 181-213.

Thomas, D. (2002). Nation-building, propaganda, and literature in Francophone Africa. Bloomington, Estados Unidos: Imprenta de la Universidad de Indiana.

Williams, R. (1994). Sociología de la cultura. Barcelona, España: Ediciones Paidós Ibérica. 